\title{
MacroH2A in stem cells: a story beyond gene
} repression

The importance of epigenetic mechanisms is most clearly illustrated during early development when a totipotent cell goes through multiple cell fate transitions to form the many different cell types and tissues that constitute the embryo and the adult. The exchange of a canonical H2A histone for the 'repressive' macroH2A variant is one of the most striking epigenetic chromatin alterations that can occur at the level of the nucleosome. Here, we discuss recent data on macroH2A in zebrafish and mouse embryos, in embryonic and adult stem cells and also in nuclear reprogramming. We highlight the role of macroH $2 \mathrm{~A}$ in the establishment and maintenance of differentiated states and we discuss its still poorly recognized function in transcriptional activation.

\section{KEYWORDS: chromatin differentiation embryonic development epigenetics histone variants macroH2A stem cells}

\section{The chromatin of pluripotent cells}

In 1938, 5 years before DNA was identified as the carrier of genetic information, Conrad Waddington had already defined epigenetics as the "science concerned with the causal analysis of development" [1]. As knowledge about the role and structure of chromatin increased, Adrian Bird redefined epigenetics as "the structural adaptation of chromosomal regions so as to register, signal or perpetuate altered activity states" [2] due to the fact that such epigenetic mechanisms constitute a level of regulation that can translate the same genotype in many different phenotypes. The pertinance of this statement is illustrated in embryonic development, when a single totipotent zygote gives rise to more than 200 specialized cell types that collectively form the embryo. As early embryonic totipotent cells divide, they undergo several cell fate transitions with progressive loss of differentiation potential until they reach their terminally differentiated state. It is very important to remember that not all cells terminate differentiation at once, in fact, all tissues retain a number of specialized stem cells with restricted potency that control tissue homeostasis and allow wounds to heal during adult life.

The genetic content of cells does not usually change as cells go through cell fate transitions during embryogenesis. Instead, how DNA is packed into chromatin and expressed during embryonic development and differentiation is regulated by epigenetic mechanisms, which are important in determining and maintaining cell identity. A large body of work has identified enzymes that modify chromatin and proteins that can read these alterations [3]. Embryonic stem (ES) cells, isolated from the inner cell mass of the blastocyst, have been extensively used as a model to study the molecular basis of epigenetic mechanisms. ES cells are pluripotent cells [4]. They can self-renew endlessly while retaining the ability to differentiate into all somatic cell types, which allows researchers to direct them into particular lineages using established protocols [5]. The ability to self-renew and to maintain the capacity to differentiate into all three germ layers is controlled by three transcription factors: Oct4, Sox 2 and Nanog [6-8] that form the core of a larger hierarchical transcriptional network $[9,10]$. In a recursive self-reinforcing circuit these core transcription factors maintain their expression at a high level in self-renewing ES cells. In this state, they directly bind and repress differentiation genes [11]. Oct 4 and Nanog are downregulated as differentiation programs are initiated. On differentiation, chromatin compacts from an initially overall dispersed distribution into localized dense regions. Early on, this observation led to the suggestion that lineage specification might be accompanied and perhaps driven by epigenetic mechanisms that lock genes regulating alternative fates into inactive chromatin domains.

\section{A histone variant implicated in development}

A plethora of post-translational histone modifications occur at the level of the building block of chromatin, the nucleosome $[12,13]$. In
Catherine Creppe, Melanija Posavec, Julien Douet

\& Marcus Buschbeck* Institute for Predictive \& Personalized Medicine of Cancer (IMPPC), Crta. Can Ruti, Cami de les Escoles, 08916 Badalona, Barcelona, Spain *Author for correspondence: Tel.: +34935543065 mbuschbeck@imppc.org 
combination with direct modification of the DNA, primarily cytosine methylation [14], histone modifications provide the molecular puzzle pieces of the still incomplete picture of how epigenetic mechanisms operate at the molecular level. The regulated exchange of canonical histones for variant proteins further adds to the modularity of the nucleosome [15]. Here, we discuss the role of the histone variant macroH2A in early development in light of novel findings in mouse ES cells. The current state of knowledge about other histone variants has recently been reviewed elsewhere [16].

Among all histone variants, macroH2A differs most from its canonical counterpart since it possesses an additional extranucleosomal macrodomain $[17,18]$. As such the incorporation of a macroH2A variant can be considered as one of the most extensive chromatin alterations occurring at the nucleosome level. In the mouse, there are two genes: macroH2A1 and macroH2A2. Since macroH $2 A 1$ can be further alternatively spliced, cells can contain three different macroH2A proteins that are distinguished as macroH2A1.1, macroH2A1.2 and macroH2A2 [17,19-21]. We will point out isoform-specific observations whenever possible. But when no distinction can be made, we will collectively refer to all three of them as macroH2A and to the two splice variants as macroH2A1. After fertilization, macroH2A1 is evicted from the maternal genome, and later, gradually reappears coinciding with the

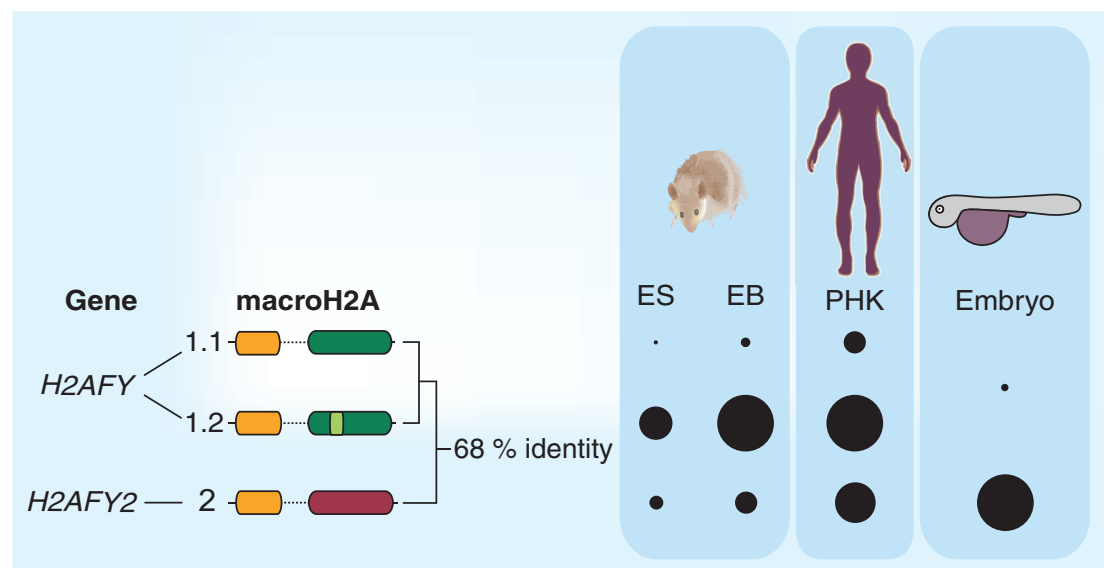

Figure 1. MacroH2A levels correlate with the state of differentiation. Overview of the macroH2A genes and proteins and their relative expression represented by the surface of the circle. Relative expression levels in cells of the same species (mouse, human and zebrafish) were determined by quantitative RT-PCR using equimolar reference samples $[27,28]$. Please note that expression levels between different species could not be directly compared and were estimated. Levels of macroH2A1.1 and macroH2A1.2 in zebrafish were not distinguished. The shared macroH2A domain structure is schematically represented (from left to right: H2A histone-fold, linker and macrodomain).

EB: Embryoid body; ES: Embryonic stem cell; PHK: Primary human keratinocyte. moment when cells start lineage-specification after the eight-cell stage [22]. It is interesting to note that this expression pattern is maintained during early embryogenesis even when macroH2A1 is expressed as a constitutively active transgene [23]. It is thought that the absence of macroH2A until the eight-cell stage is required for normal embryonic development because the tethering of macroH2A to chromatin beyond this stage by addition of peptide that mediates stage-specific H2AX diposition impaired early embryogenesis [23].

In ES cells, macroH2A is expressed at moderate levels and its expression increases at differentiation $[24,25]$. The end points of the differentiation induced upregulation of macroH2A can be nicely illustrated by comparing macroH2A1 levels in mouse ES cells with liver tissue (Figure 1), which contains the maximal amount of macroH2A estimated to reach $3 \%$ of total $\mathrm{H} 2 \mathrm{~A}$ [26]. In self-renewing E14 mouse ES cells the predominantly expressed form of macroH2A is macroH2A1.2, which constitutes approximately $85 \%$ of the cellular macroH2A protein [27]. In the same cells, macroH2A 2 is expressed at much lower levels and macroH2A1.1 is undetectable. Analysis of more than 40 different established human and murine cell lines demonstrated that macroH2A1.2 generally tends to be expressed at higher levels than macroH2A2 and macroH2A1.1 [Valero V and Buschbeck M, Unpublished DATA] suggesting that this expression pattern is not specific for ES cells but possibly a general characteristic of proliferating cells. This rule would have at least one exception. Early zebrafish embryos exclusively express the macroH $2 A 2$ ortholog while macroH2A1 is not detected [28]. Knockdown of macroH2A2 in zebrafish embryos provoked severe developmental defects, including malformations of the body structure and brain [28]. Importantly, some of these defects could be rescued by expressing human macroH2A2 [28]. This observation provided the first strong evidence for an important role of macroH2A proteins in differentiation and developmental processes. This is in contrast to the mouse, in which knockout of macroH $2 \mathrm{Al}$ has been reported to display only mild phenotypes [29,30]. The exciting discrepancy between mice and fish could be due to different reasons, of which the most intuitive would be a direct functional compensation by the remaining macroH2A2. Other possible reasons could lie in different isoform-specific functions of macroH2A 1 and macroH2A 2 or in the difference in the duration of the embryonic developmental 
process, which could allow for compensation by alternative mechanisms in the slower developing mouse embryo but not in the faster developing zebrafish. A wider analysis of loss-of-function phenotypes and the identification of operating compensatory mechanisms will be required before we can fully appreciate the extension and the limits of macroH2A's function in developmental regulation.

\section{MacroH2A \& the differentiation of ES cells}

Functional redundancy between macroH2A1 and macroH2A 2 has been proposed as an explanation for the lack of defects in mouse mutant embryos. We decided to use E14 mouse ES cells that predominantly express macroH2A1.2 to approach the function of macroH2A in early lineage specification. It is noteworthy that shRNA mediated knockdown of macroH2A1 did not affect ES cell proliferation, survival or self-renewal [27,31]. However, differences between macroH2A1-deficient and control cells became obvious when differentiation was induced using three different approaches: neuronal differentiation, multilineage differentiation in embryoid bodies and in vivo differentiation in xenografted teratomas [27]. Under all three conditions macroH2A1-deficiency partially impaired the differentiation capacity of the ES cells. This was reflected in the expression level of differentiation genes whose partial inhibition came in two flavors: some genes did not reach the normal amplitude of activation while others were delayed. In addition, macroH2A1-deficient ES cells retained abnormally high-expression levels of the stem cell markers Nanog, Oct 4 and Sox 2 after prolonged induction of differentiation [27]. It is interesting to point out that a similar phenotype has also been seen when other well established epigenetic regulators such as G9a and DNMT3a/b were depleted $[32,33]$.

The commitment defect was also seen in teratomas derived from macroH2A1-knockdown cells that displayed a massive expansion of undifferentiated carcinoma cells [27]. We conclude that macroH2A is required for the proper execution of differentiation programs during ES cell differentiation. This conclusion differs from that obtained by Tansijevic and Rasmussen, who reported that double macroH2A1 and macroH2A2 deficiency in ES cells did not prevent $\mathrm{X}$ chromosome inactivation and the formation of embryoid bodies [31]. We believe that this discrepancy could be the result of differences in genetic background or in the quantitative nature and timing of the analyses performed. Ultimately, genetic ablation of macroH $2 A s$ in ES cells with different genetic backgrounds will be required.

In support of a role of macroH2A in differentiation, macroH2A is rapidly removed from chromatin during reprogramming by nuclear transfer [34]. A recent study demonstrated the relevance of this observation by demonstrating that the knockdown of macroH2A in differentiated cells facilitated their reprogramming and the reactivation of Oct4 and Sox2 [35]. Gurdon et al. noted earlier that different cell types had different intrinsic resistance to reprogramming by nuclear transfer [36]. Comparing susceptible mouse epiblast-derived stem cells with resistant embryonic fibroblasts they were able to identify macroH2A as one of the epigenetic layers that confers resistance to reprogramming [35]. The same authors concluded that it is likely that the observed resistance directly reflects the stability of the differentiated state and that macroH2A contributes to this stability [37]. Taking these results together with our observations in mouse ES cells, we postulate that macroH2A is involved in the establishment of the stable epigenomes of differentiated cells as well as in their maintenance. In line with this, macroH2A1 levels also correlated with the degree of differentiation in human skin sections and in multilayered colonies formed by primary human keratinocytes [27]. The ability of these keratinocytes to form $3 \mathrm{D}$ colonies that differentiate from bottom to top can be used to assess the number of competent tissue stem cells in the heterogeneous primary culture. Overexpression of macroH2A1 reduced the number of colonyforming cells, while shRNA-mediated repression of macroH2A1 increased it [27]. From these observations, we conclude that macroH2A levels modulate the intricate balance between selfrenewal and differentiation not only of ES cells but also of adult stem cells.

\section{Is macroH2A also a gene 'activator'?}

The early finding that macroH2A was enriched on the inactive $\mathrm{X}$ chromosome in female cells suggested a repressive function [38]. Subsequently, this has been well established in vitro and in vivo by a large body of evidence (reviewed in $[15,18]$ ). In mouse liver chromatin, for instance, macroH $2 \mathrm{~A} 1$ was found to be depleted from the transcribed regions of most active genes [39,40]. Two other genome-wide studies later confirmed in other cell types that macroH2A occupancy had a general negative correlation with transcriptional 
activity $[28,41]$. Both studies, however, also pointed out that a small number of genes are expressed while harboring macroH2A nucleosomes on the promoter and/or the transcription start site proximal transcribed region. Moreover, Gamble et al. further demonstrated that macroH2A is required for inducible activation of a set of target genes [41], thereby corroborating and extending on the earlier observation that macroH2A1.1 was required for the heat shock-mediated activation of Hsp70.1 [42]. In addition, many of the differentiation genes that were not fully activated in macroH2A1-deficient ES cells were also enriched for macroH2A in undifferentiated cells. This suggests that macroH2A could exert a similar prosignal sensitivity and proactivation function in ES cells [27]. In HeLa cells macroH2A1.1specific recruitment of parylated PARP1 and its inhibition under normal growth conditions was required for maintaining the $H s p 70.1$ gene sensitive to heat shock [42]. In ES cells, which lack

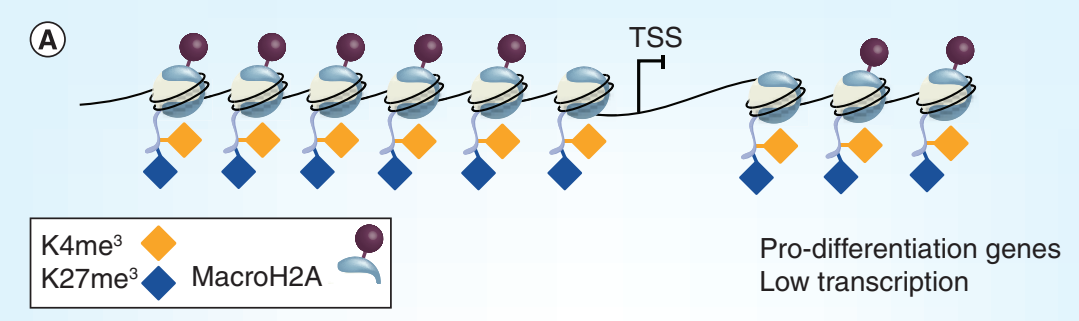

(B)

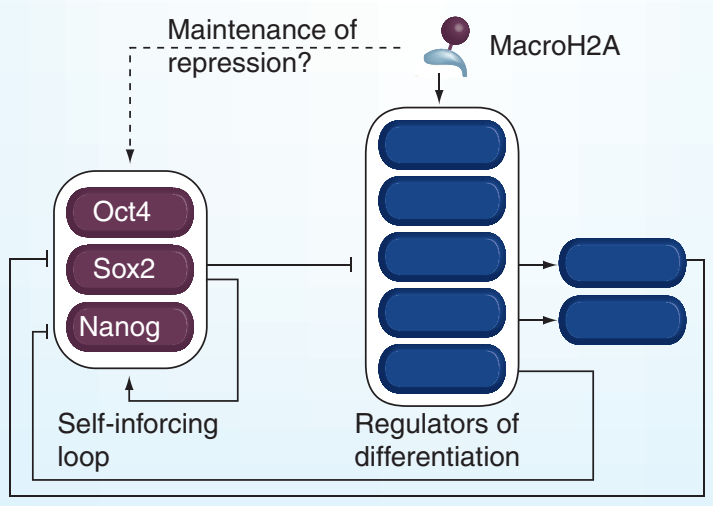

Negative feedback loops

Figure 2. MacroH2A interacts with the regulatory circuits of stem cell fate. (A) Differentiation genes are marked by macroH2A and bivalent lysine 4 and 27 methylations on histone $\mathrm{H} 3$. This chromatin state keeps regulatory differentiation genes repressed but highly sensitive to activating cues. (B) Strongly simplified figure illustrating how macroH2A could interfere with the regulatory circuits of ES cells. The core pluripotency transcription factors reinforce their own expression and repress regulatory differentiation genes. When external signals overcome this repression, the products of differentiation genes such as Gata6 and $\mathrm{Cd} \times 2$ contribute to the inactivation of the core transcription factors through direct and indirect negative feedback loops, thereby sealing off the cell's commitment to differentiation. The presence of macroH2A on differentiation genes facilitates their activation by lowering the activation threshold. For more detailed information on how these regulatory circuits work please see the review by Niwa [11].

TSS: Transcription start site.
macroH2A1.1 the underlying molecular mechanism still remains elusive. But we speculate that macroH2A proteins either directly or through interacting partners stabilize chromatin states that tend to be repressed but at the same time are highly sensitive to signal-induced activation. This working model reconciles the newly recognized proactivation functions of macroH2A with its well established role as repressor.

In ES cells such a chromatin state has been well described for many differentiation regulatory genes that were termed bivalent genes due to the presence of the apparently opposing lysine 4 and 27 trimethylation marks on histone $\mathrm{H} 3[43,44]$. As illustrated in Figure 2, many of the macroH2A target genes are marked by 'opposing' lysine 4 and 27 trimethylation marks on histone H3 [27]. Interestingly, in self-renewing ES cells loss of macroH2A did not affect the levels of the two bivalent lysine methylations on genes that displayed a clear defect in their differentiation-induced activation. Thus, our data now suggest that macroH2A is required for proper functionality of bivalent genes without affecting the bivalent signature per se.

How can we put the new knowledge about macroH2A into context of what is known about the regulation of pluripotency in ES cells? Although it is clear that the core transcription factors Oct4, Nanog and Sox 2 self-sustain their expression, it has also been shown that induction of differentiation genes exerts negative feedback on their expression (reviewed in [11]). For instance, both $\mathrm{Cdx} 2$ and Gata6 were shown to directly and indirectly repress the Pouff1/Oct4 and Nanog genes. Our data now suggest that the presence of macroH2A on prodifferentiation genes lowers the threshold for their activation and thus, through such a negative feedback loop, indirectly contributes to the repression of the core transcription factors. Considering the repressive potential of macroH2A also provokes the question of whether macroH2A could also directly contribute to the repression of the pluripotency core transcription factors. However, the fact that defects in the expression of differentiation genes induced by loss of macroH2A1 preceded the defective repression of pluripotency genes argues against this hypothesis and in favor of an indirect mechanism. Even if macroH2A might not be required for the establishment of the repressed chromatin state on pluripotency genes, presently we cannot exclude that it might be involved in its maintenance [45], as suggested by Pasque et al. [37].

Although macroH2A is a well-characterized component of repressed chromatin, its regulatory 
role in differentiation relies at least partially on its much less understood function in signalinduced gene activation. It will be interesting to see whether macroH2A also affects the selfrenewal and differentiation of human ES cells, which depend on other signals for self-renewal compared with mouse ES cells [46]. Another important question is whether the repression of macroH2A could help in the generation of human induced pluripotent cells for purposes of regenerative medicine.

\section{Future perspective}

MacroH2A levels correlate with differentiation or - if one prefers - inversely correlates with cellular potency. Increasing macroH2A levels affects the balance between self-renewal and differentiation by facilitating the activation of its target genes that are part of transcriptional differentiation programs. MacroH2A contributes to the establishment but also the stable maintenance of differentiated phenotypes.

These conclusions are based on the limited amount of data available today. Future work will be required to test whether these statements prove right in general or only for a limited number of plastic cell types and their differentiation processes. But it is beyond doubt that with the characterization of the first physiological functions of macroH2A in zebrafish development and ES cell differentiation, research on this unique histone variant has reached an exciting turning point. We are now in an unique position to analyze the functional relevance of its exceptional domain structure as well as suggested protein [18] and metabolite interactions $[47,48]$. Virtually nothing is known about how the incorporation of macroH2A is regulated. In this context it is interesting to point that - like most histone - macroH2A undergoes several post-translational modifications [49-51]. Further analysis of the functional consequences of these modifications holds the promise to provide important clues. It is further interesting to point out that macroH2A might have an ambivalent function in chromatin condensation. In vitro the linker domain of macroH2A is sufficient to mediate a histone H1-like condensation of nucleosome arrays [52]. However, the presence of the adjacent macro domain inhibits this function. This suggests that macroH2A-containing chromatin might be a metastable form of open chromatin, whose condensation could be induced by factors altering macroH2A protein structure.

Bernstein et al. further fueled the interest in macroH2A by showing that its loss contributed to the progression of melanoma to the advanced and metastatic state [53]. In this context, it is interesting to point out that melanoma cells are dedifferentiated cells that reactivate embryonic signaling pathways [54]. Others could point out functional differences between macroH2A1 splice variants in different types of cancer $[55,56]$. Ongoing and future studies are likely to reveal the importance, the regulation and the molecular function of macroH2A-containing epigenetic mechanisms in a larger number of physiological and pathological processes. The door is wide open for new discoveries.

\section{Acknowledgements}

The authors would like to thank V Pasque for his comments on the manuscript.

\section{Executive summary}

\section{The chromatin of pluripotency}

- Embryonic stem (ES) cells are regulated by regulatory circuits of transcription factors.

- ES cells have an open chromatin structure.

\section{A histone variant implicated in development}

- MacroH2A is considered a repressive histone variant.

- It is essential for proper zebrafish development.

\section{MacroH2A \& the differentiation of ES cells}

- MacroH2A levels correlate with the state of differentiation.

- MacroH2A affects the balance between self-renewal and differentiation of ES cells by facilitating lineage commitment.

- MacroH2A contributes to the establishment but also the stable maintenance of differentiated phenotypes.

- In addition to ES cells, macroH2A also regulates the tissue stem cells of the skin.

Is macroH2A also a gene 'activator'?

- In ES cells, macroH2a targets bivalent differentiation genes and facilitates their activation.

- Thereby, macroH2A influences the regulatory circuits of ES cells.

- This observation provided the physiological relevance of its poorly recognized function in transcriptional activation. 


\section{Financial \& competing interests disclosure}

Research in the Buschbeck laboratory is supported by Spanish MICINN grants (SAF2009-08496 and RYC2010-07337). C Creppe holds a postdoctoral FEBS fellowship, J Douet a postdoctoral Beatriu de Pinós contract (AGAUR), M Posavec a predoctoral FI fellowship (AGAUR) and M Buschbeck is a Ramón Y Cajal fellow (MICINN). The authors have no other relevant affiliations or financial involvement with any organization or entity with a financial interest in or financial conflict with the subject matter or materials discussed in the manuscript apart from those disclosed.

No writing assistance was utilized in the production of this manuscript.

\section{References}

Papers of special note have been highlighted as:

- of interest

"II of considerable interest

1 Waddington $\mathrm{CH}$. The Epigenetics of Birds. Cambridge University Press, Cambridge, UK (1952).

2 Bird A. Perceptions of epigenetics. Nature 447(7143), 396-398 (2007).

3 Bannister AJ, Kouzarides T. Regulation of chromatin by histone modifications. Cell Res. 21(3), 381-395 (2011).

4 Evans M. Discovering pluripotency: 30 years of mouse embryonic stem cells. Nat. Rev. Mol. Cell Biol. 12(10), 680-686 (2011).

5 Smith AG. Embryo-derived stem cells: of mice and men. Annu. Rev. Cell Dev. Biol. 17, 435-462 (2001).

6 Boyer LA, Lee TI, Cole MF et al. Core transcriptional regulatory circuitry in human embryonic stem cells. Cell 122(6), 947-956 (2005).

- Leading work among the manuscripts that reported the identification of the trancriptional circuit that maintains pluripotency.

7 Chew JL, Loh YH, Zhang W et al. Reciprocal transcriptional regulation of Pou5f1 and Sox 2 via the Oct $4 /$ Sox 2 complex in embryonic stem cells. Mol. Cell Biol. 25(14), 6031-6046 (2005).

8 Loh YH, Wu Q, Chew JL et al. The Oct4 and Nanog transcription network regulates pluripotency in mouse embryonic stem cells. Nat. Genet. 38(4), 431-440 (2006).

9 Chen X, Xu H, Yuan P et al. Integration of external signaling pathways with the core transcriptional network in embryonic stem cells. Cell 133(6), 1106-1117 (2008).

10 Kim J, Chu J, Shen X, Wang J, Orkin SH. An extended transcriptional network for pluripotency of embryonic stem cells. Cell 132(6), 1049-1061 (2008).

11 Niwa $\mathrm{H}$. How is pluripotency determined and maintained? Development 134(4), 635-646 (2007).

12 Hake SB, Xiao A, Allis CD. Linking the epigenetic 'language' of covalent histone modifications to cancer. Br. J. Cancer 96(Suppl.), R31-R39 (2007).

13 Kouzarides T. Chromatin modifications and their function. Cell 128(4), 693-705 (2007).

14 Williams K, Christensen J, Helin K. DNA methylation: TET proteins-guardians of $\mathrm{CpG}$ islands? EMBO Rep. 13(1), 28-35 (2011).

15 Boulard M, Bouvet P, Kundu TK, Dimitrov $S$. Histone variant nucleosomes: structure, function and implication in disease. Subcell Biochem. 41, 71-89 (2007).

16 Banaszynski LA, Allis CD, Lewis PW. Histone variants in metazoan development. Dev. Cell 19(5), 662-674 (2011).

17 Pehrson JR, Fried VA. MacroH2A, a core histone containing a large nonhistone region. Science 257(5075), 1398-1400 (1992).

18 Buschbeck M, Di Croce L. Approaching the molecular and physiological function of macroH2A variants. Epigenetics 5(2), 118-123 (2010).

19 Rasmussen TP, Huang T, Mastrangelo MA, Loring J, Panning B, Jaenisch R. Messenger RNAs encoding mouse histone macroH $2 \mathrm{~A} 1$ isoforms are expressed at similar levels in male and female cells and result from alternative splicing. Nucleic Acids Res. 27(18), 3685-3689 (1999).

20 Chadwick BP, Willard HF. Histone H2A variants and the inactive $\mathrm{X}$ chromosome: identification of a second macroH $2 \mathrm{~A}$ variant. Hum. Mol. Genet. 10(10), 1101-1113 (2001).

21 Costanzi C, Pehrson JR. MACROH2A2, a new member of the MARCOH2A core histone family. J. Biol. Chem. 276(24), 21776-21784 (2001).

22 Chang CC, Ma Y, Jacobs S, Tian XC, Yang $\mathrm{X}$, Rasmussen TP. A maternal store of macroH2A is removed from pronuclei prior to onset of somatic macroH2A expression in preimplantation embryos. Dev. Biol. 278(2), 367-380 (2005).

23 Nashun B, Yukawa M, Liu H, Akiyama T, Aoki F. Changes in the nuclear deposition of histone $\mathrm{H} 2 \mathrm{~A}$ variants during pre-implantation development in mice. Development 137(22), 3785-3794 (2010).

- Provides evidence that a tight regulation of macroH2A levels is essential at the premorula stage of early development.

24 Pehrson JR, Costanzi C, Dharia C. Developmental and tissue expression patterns of histone macroH2A1 subtypes. J. Cell Biochem. 65(1), 107-113 (1997).

25 Dai B, Rasmussen TP. Global epiproteomic signatures distinguish embryonic stem cells from differentiated cells. Stem Cells 25(10), 2567-2574 (2007).

26 Mietton F, Sengupta AK, Molla A et al. Weak but uniform enrichment of the histone variant macroH2A 1 along the inactive X chromosome. Mol. Cell Biol. 29(1), 150-156 (2009).

27 Creppe C, Janich P, Cantariño N et al. $\mathrm{MacroH} 2 \mathrm{~A}$ regulates the commitment to differentiation of embryonic and adult stem cells. Mol. Cell Biol. doi:10.1128/ MCB.06323-11 (2012) (Epub ahead of print).

- Demonstrates that the proactivating function of macroH2A contributes to the regulation of embryonic stem cell differentiation.

28 Buschbeck M, Uribesalgo I, Wibowo I et al. The histone variant macroH $2 \mathrm{~A}$ is an epigenetic regulator of key developmental genes. Nat. Struct. Mol. Biol. 16(10), 1074-1079 (2009).

"- This study provided the first proof for a role of macroH $2 \mathrm{~A}$ in development and differentiation.

29 Changolkar LN, Costanzi C, Leu NA, Chen D, McLaughlin KJ, Pehrson JR. Developmental changes in histone macroH2A1-mediated gene regulation. Mol. Cell Biol. 27(7), 2758-2764 (2007).

- Together with [30], this work provides the description of the metabolic phenotype of macroH2A1-deficient mice.

30 Boulard M, Storck S, Cong R, Pinto R, Delage $\mathrm{H}$, Bouvet $\mathrm{P}$. Histone variant macroH2A1 deletion in mice causes female-specific steatosis. Epigenetics Chromatin 3(1), 8 (2010).

- Together with [29], this work provides the description of the metabolic phenotype of macroH2A1-deficient mice.

31 Tanasijevic B, Rasmussen TP. X chromosome inactivation and differentiation occur readily in ES cells doubly-deficient for macroH2A1 and macroH2A2. PLoS ONE 6(6), e21512 (2011).

32 Epsztejn-Litman S, Feldman N, AbuRemaileh $\mathrm{M}$ et al. De novo DNA methylation promoted by G9a prevents reprogramming of embryonically silenced genes. Nat. Struct. Mol. Biol. 15(11), 1176-1183 (2008).

33 Feldman N, Gerson A, Fang J et al. G9a-mediated irreversible epigenetic inactivation of Oct-3/4 during early embryogenesis. Nat. Cell Biol. 8(2), 188-194 (2006). 
34 Chang CC, Gao S, Sung LY et al. Rapid elimination of the histone variant $\mathrm{MacroH} 2 \mathrm{~A}$ from somatic cell heterochromatin after nuclear transfer. Cell Reprogram. 12(1), 43-53 (2010).

35 Pasque V, Gillich A, Garrett N, Gurdon JB. Histone variant macroH $2 \mathrm{~A}$ confers resistance to nuclear reprogramming. EMBO J. 30(12), 2373-2387 (2011).

"- Demonstrates that macroH2A maintains differentiated states and constitutes a layer of resistance to reprogramming. Pasque V, Jullien J, Miyamoto K, Halley-Stott RP, Gurdon JB. Epigenetic factors influencing resistance to nuclear reprogramming. Trends Genet. 27(12), 516-525 (2011).

37 Pasque V, Halley-Stott RP, Gillich A, Garrett N, Gurdon JB. Epigenetic stability of repressed states involving the histone variant macroH2A revealed by nuclear transfer to xenopus oocytes. Nucleus 2(6), 533-539 (2011).

38 Costanzi C, Pehrson JR. Histone macroH2A1 is concentrated in the inactive $\mathrm{X}$ chromosome of female mammals. Nature 393(6685), 599-601 (1998).

39 Changolkar LN, Pehrson JR. macroH2A1 histone variants are depleted on active genes but concentrated on the inactive X chromosome. Mol. Cell Biol. 26(12), 4410-4420 (2006).

40 Changolkar LN, Singh G, Cui Ket al. Genome-wide distribution of macroH2A1 histone variants in mouse liver chromatin. Mol. Cell Biol. 30 (23), 5473-5483 (2010).

41 Gamble MJ, Frizzell KM, Yang C, Krishnakumar R, Kraus WI. The histone variant macroH2A1 marks repressed autosomal chromatin, but protects a subset of its target genes from silencing. Genes Dev. 24(1), 21-32 (2010).

- Together with [42], this article broke the dogma of macroH2A as a sole repressor.

42 Ouararhni K, Hadj-Slimane R, Ait-Si-Ali S et al. The histone variant mH2A1.1 interferes with transcription by down-regulating PARP-1 enzymatic activity. Genes Dev. 20(23), 3324-3336 (2006).

- Together with [41], this article broke the dogma of macroH2A as a sole repressor.

43 Azuara V, Perry P, Sauer S et al. Chromatin signatures of pluripotent cell lines. Nat. Cell Biol. 8(5), 532-538 (2006).

44 Bernstein BE, Mikkelsen TS, Xie X et al. A bivalent chromatin structure marks key developmental genes in embryonic stem cells. Cell 125(2), 315-326 (2006).

45 Heard E. Delving into the diversity of facultative heterochromatin: the epigenetics of the inactive X chromosome. Curr. Opin. Genet. Dev. 15(5), 482-489 (2005).

46 Silva J, Smith A. Capturing pluripotency. Cell 132(4), 532-536 (2008).

- Short review raising and discussing some of the most essential questions in embryonic stem cell biology.

47 Kustatscher G, Hothorn M, Pugieux C, Scheffzek K, Ladurner AG. Splicing regulates NAD metabolite binding to histone macroH2A. Nat. Struct. Mol. Biol. 12(7), 624-625 (2005).

48 Timinszky G, Till S, Hassa PO et al. A macrodomain-containing histone rearranges chromatin upon sensing PARP1 activation. Nat. Struct. Mol. Biol. 16(9), 923-929 (2009).

49 Bernstein E, Muratore-Schroeder TL, Diaz RL et al. A phosphorylated subpopulation of the histone variant macroH2A1 is excluded from the inactive $\mathrm{X}$ chromosome and enriched during mitosis. Proc. Natl Acad. Sci. USA 105(5), 1533-1538 (2008).

50 Abbott DW, Chadwick BP, Thambirajah AA, Ausio J. Beyond the Xi: macroH2A chromatin distribution and post-translational modification in an avian system. J. Biol. Chem. 280(16), 16437-16445 (2005).

51 Thambirajah AA, Li A, Ishibashi T, Ausio J. New developments in post-translational modifications and functions of histone $\mathrm{H} 2 \mathrm{~A}$ variants. Biochem. Cell Biol. 87(1), 7-17 (2009).

52 Muthurajan UM, McBryant SJ, Lu X, Hansen JC, Luger K. The linker region of macroH2A promotes self-association of nucleosomal arrays. J. Biol. Chem. 286(27), 23852-23864 (2011).

53 Kapoor A, Goldberg MS, Cumberland LK et al. The histone variant macroH2A suppresses melanoma progression through regulation of CDK8. Nature 468(7327), 1105-1109 (2010).

"- Delivered the first proof that loss of macroH2A is involved in cancer progression.

54 Strizzi L, Hardy KM, Kirsammer GT, Gerami P, Hendrix MJ. Embryonic signaling in melanoma: potential for diagnosis and therapy. Lab. Invest. 91(6), 819-824 (2011).

55 Novikov L, Park JW, Chen H, Klerman H, Jalloh AS, Gamble MJ. QKI-mediated alternative splicing of the histone variant macroH2A1 regulates cancer cell proliferation. Mol. Cell Biol. 31(20), 4244-4255 (2011).

56 Sporn JC, Kustatscher G, Hothorn T et al. Histone macroH2A isoforms predict the risk of lung cancer recurrence. Oncogene 28(38), 3423-3428 (2009). 